Article

\title{
Synthesis of Poly(methyl methacrylate) Grafted Multiwalled Carbon Nanotubes via a Combination of RAFT and Alkyne-Azide Click Reaction
}

\author{
Wenzhong Ma ${ }^{1, * \mathbb{D}}$, Yuchen Zhao ${ }^{1}$, Zhiwei Zhu ${ }^{1}$, Lingxiang Guo ${ }^{1}$, Zheng Cao ${ }^{1}$, Yanping Xia ${ }^{1}$, \\ Haicun Yang ${ }^{1}$, Fanghong Gong ${ }^{1}$ and Jing Zhong ${ }^{2}$ \\ 1 Jiangsu Key Laboratory of Environmentally Friendly Polymeric Materials, School of Materials Science and \\ Engineering, Jiangsu Collaborative Innovation Center of Photovoltaic Science and Engineering, \\ Changzhou University, Changzhou 213164, Jiangsu, China; zhaoyuchentmac@foxmail.com (Y.Z.); \\ zedzzw@gmail.com (Z.Z.); glx16441216@gmail.com (L.G.); zcao@cczu.edu.cn (Z.C.); \\ xiayanping0715@126.com (Y.X.); yhcbobo@cczu.edu.cn (H.Y.); fhgong@cczu.edu.cn (F.G.) \\ 2 Jiangsu Key Laboratory of Advanced Catalytic Materials and Technology, School of Petrochemical \\ Engineering, Changzhou University, Changzhou 213164, Jiangsu, China; zjwyz@cczu.edu.cn \\ * Correspondence: wenzhong-ma@cczu.edu.cn; Tel.: +86-519-86330095
}

Received: 28 December 2018; Accepted: 4 February 2019; Published: 12 February 2019

\begin{abstract}
An efficient synthesis route was developed for the preparation of multiwalled carbon nanotube (MWCNT) nanohybrids using azide-terminated poly(methyl methacrylate) (PMMA) via a combination of reversible addition fragmentation chain transfer (RAFT) and the click reaction. A novel azido-functionalized chain transfer agent $\left(\mathrm{DMP}-\mathrm{N}_{3}\right)$ was prepared and subsequently employed to mediate the RAFT polymerizations of methyl methacrylate (MMA). The RAFT polymerizations exhibited first-order kinetics and a linear molecular weight dependence with the conversion. The kinetic results show that the grafting percentage of PMMA on the MWCNTs surface grows along with the increase of the reaction time. Even at $50{ }^{\circ} \mathrm{C}$, the grafting rate of azide-terminated PMMA is comparatively fast in the course of the click reaction, with the alkyne groups adhered to MWCNTs in less than $24 \mathrm{~h}$. The successful functionalization of PMMA onto MWCNT was proved by FTIR, while TGA was employed to calculate the grafting degree of PMMA chains (the highest $\mathrm{GP}=21.9 \%$ ). Compared with the pristine MWCNTs, a thicker diameter of the MWCNTs-g-PMMA was observed by TEM, which confirmed the grafted PMMA chain to the surface of nanotubes. Therefore, the MWCNTs- $g$-PMMA could be dispersed and stably suspended in water.
\end{abstract}

Keywords: multi-wall carbon nanotube (MWCNT); azide-alkyne click chemistry; RAFT polymerization; PMMA

\section{Introduction}

Nanoscience and nanotechnology have brought us the excellent development of many novel categories of functional materials and have become remarkable fields of study [1,2]. Recently, carbon nanotubes (CNTs) have acquired increasing importance and popularity in membrane science and technology due to their high permeability and selectivity, which they owe to the rapid flux through the hollow interior and nano-scale diameter of CNTs [3-7]. For instance, multiwalled carbon nanotubes (MWCNTs) with outer diameters $(2-100 \mathrm{~nm}$ ) exhibit a significantly high permeability in membrane process applications because of the large surface area [6]. The MWCNT hybrid nanostructure and composite materials with the introduction of polymer chemistry have dramatically attracted attention [8,9]. These nanocomposite materials complement the characteristics of functional polymers and thus provide improved nano-scale dispersing, hydrophilicity, electric properties, etc. [10]. Some 
researchers have directly immobilized MWCNTs into a polymeric membrane by the blending method due to its easy manipulation and mild conditions [11,12]. However, despite the outstanding properties of MWCNT composite materials, the tendency to be polymerized caused by the big inherent van der Waals forces of MWCNTs restricts its application to the fabrication of nanocomposites. [13].

Among the surface modification method, the "grafting to" technique is one of the most convenient techniques to cap polymer chains which can adjust the dispersibility of nanoparticles in polymer matrices [14]. In this approach, functional group-terminated polymer chains can graft onto the surface of nanoparticles in a highly efficient reaction, resulting in the formation of tethered polymer chains [15]. To achieve dense polymer layers attached to the MWCNT surface, strong interactions between polymer chains and the MWCNT surface are required. Poly(methyl methacrylate) (PMMA) is usually studied as a compatibilizer agent for polymer/nanoparticle composites [16]. Recently, "click chemistry" has attracted more attention in surface modification for nanoparticles due to its high yields without byproducts [17-20]. When combined with reversible addition-fragmentation chain transfer (RAFT), the precise predesign of the molecular weight, structure, and functionality of polymers can be controlled by living polymerizations [21]. For example, Singha and co-workers synthesized a hydrophilic MWCNT based upon the Diels-Alder (DA) click reaction by one step [22]. Nonetheless, few have been reported in the field of PMMA-functioned MWCNTs, synthesized through the click reaction.

In this work, by using azide/alkyne end groups, PMMA with an azide end group can effectively graft onto the MWCNT surface. To do this, the RAFT agent 2-dodecylsulfanylthiocarbonylsulfanyl-2-methylpropionic acid 3-azidopropyl ester (DMP- $\left.\mathrm{N}_{3}\right)$ was used in the polymerization of PMMA. Then, azide-terminated PMMA was attached to the surface of alkyne-terminated MWCNTs via the "grafting to" approach. Due to the PMMA chain on the MWCNT surface, the nanohybrids can be stably dispersed in water and have potential for the preparation of MWCNT composite materials.

\section{Materials and Methods}

\subsection{Materials}

MWCNTs were obtained from Nanjing XFNANO Materials Tech Co., Ltd. (China). Methyl methacrylate (MMA) was obtained from Shanghai Chemical Plant (China). The inhibitor was removed by a basic alumina column, purified under lower pressure, and stored in an Ar atmosphere at $-5{ }^{\circ} \mathrm{C}$. Sodium azide, 4 -( $N, N$-dimethylamino)pyridine(DMAP), and 1-(3-Dimethylaminopropyl)-3ethylcarbodiimide were obtained from Aladdin Industrial Corporation (China). Azobisisobutyronitrile (AIBN) was purchased from Jiangsu Qiangsheng Chemical Co., Ltd. (China). N,N-dimethylacetamide (DMAc), propargyl alcohol, tetrahydrofuran (THF, analytical grade), and anisole (AR) were purchased from Shanghai Lingfeng Chemical Reagent Co., Ltd. (China). Thionyl chloride (analytical grade) was purchased from Sinopharm Chemical Reagent Co., Ltd. (China).

\subsection{Synthesis of Azide-Terminated Poly(methyl methacrylate) (PMMA)}

Before synthesizing azide-terminated PMMA, RAFT agent 2dodecylsulfanylthiocarbonylsulfanyl-2-methylpropionic acid 3-azidopropyl ester (DMP- $\left.\mathrm{N}_{3}\right)$ was synthesized according to the reported method [23]. FTIR analysis was performed to confirm successful azide-RAFT agent preparation, as shown in Figure 1a. FTIR $(\mathrm{KBr})$ (wavenumber, $\mathrm{cm}^{-1}$ ): 2923 (C-Cs), 2100 (C-N=N=N), 1735 (C=O), 1064 (C=S), 1250 (C-S). Figure $1 \mathrm{~b}$ shows the ${ }^{1} \mathrm{H}$ NMR spectra of DMP-N ${ }_{3}$. The peak at ${ }^{1} \mathrm{H}-\mathrm{NMR}\left(400 \mathrm{MHz}, \mathrm{CDCl}_{3}, \mathrm{TMS}\right)$ for DMP-N $\mathrm{D}_{3}(\delta, \mathrm{ppm}): 0.88(\mathrm{t}, 3 \mathrm{H}$, $\left.-\mathrm{CH}_{3}\right), 1.25\left(\mathrm{~m}, 2 \mathrm{H},-\mathrm{CH}_{2}-\right), 1.72\left(\mathrm{~s}, 6 \mathrm{H},-\mathrm{CH}_{3}\right), 1.91\left(\mathrm{~m}, 2 \mathrm{H},-\mathrm{CH}_{2}-\right), 3.35\left(\mathrm{t}, 2 \mathrm{H},-\mathrm{CH}_{2}-\right), 4.19(\mathrm{t}, 2 \mathrm{H}$, $\left.-\mathrm{CH}_{2}-\right)$. 

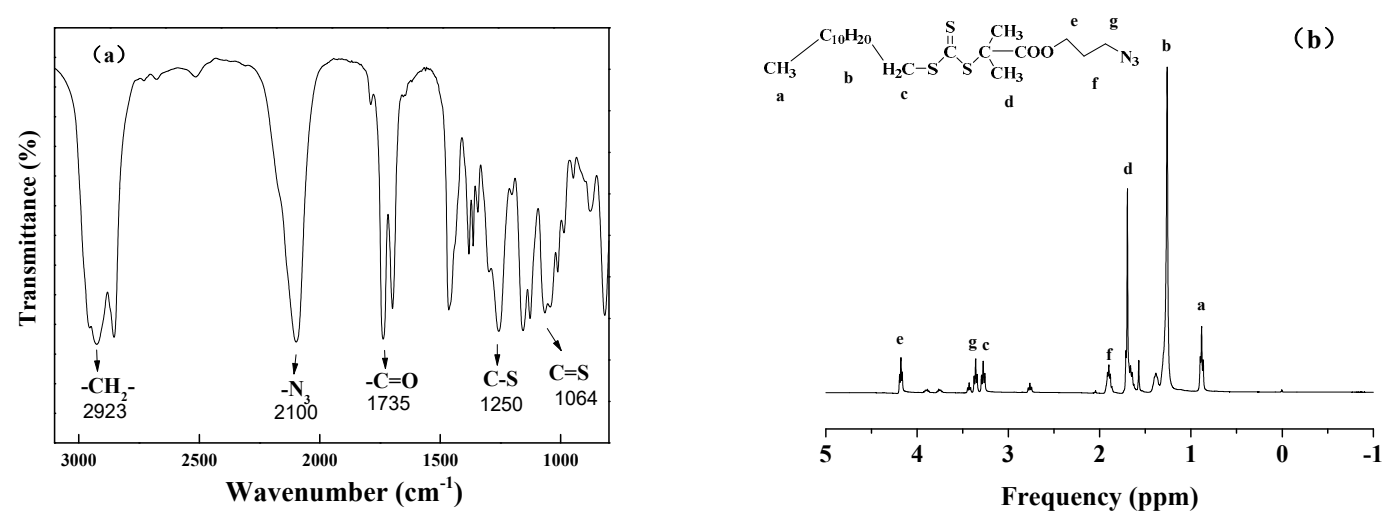

Figure 1. (a) FTIR spectra of the DMP-N 3 (b) ${ }^{1} \mathrm{H}$ spectra of the DMP-N $\mathrm{N}_{3}$.

For the typical polymerization processing of PMMA, $5 \mathrm{~g}$ of monomer MMA, chain transfer agent $\mathrm{DMP}^{-\mathrm{N}_{3}}(0.168 \mathrm{~g})$, and AIBN $(8.2 \mathrm{mg})$, which were dissolved in dried anisole $(20 \mathrm{ml})$, was added to a $50 \mathrm{ml}$ Schlenk flask. The mixture solution was degassed with nitrogen using a three-way tube (three cycles). Then, a RAFT polymerization reaction was performed at $60{ }^{\circ} \mathrm{C}$ for $10 \mathrm{~h}$. After polymerization, the azide-terminated PMMA was obtained when the unreacted MMA monomer was removed with THF for $24 \mathrm{~h}$ by Soxhlet apparatus. The resulting polymer was dried at $25{ }^{\circ} \mathrm{C}$ in a vacuum oven for $12 \mathrm{~h}$. Scheme 1 shows the route of synthesis of azide-terminated PMMA. The resulting polymer was dried at $25^{\circ} \mathrm{C}$ in a vacuum oven for $12 \mathrm{~h}$. Scheme 1 shows the route of synthesis of azide-terminated PMMA.

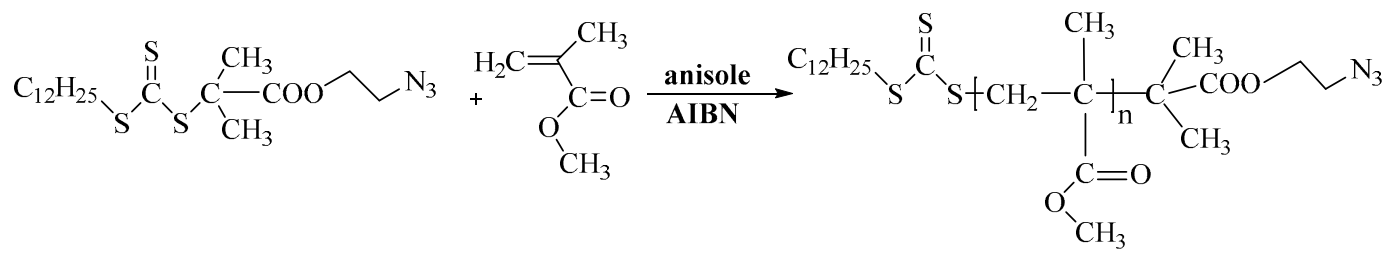

Scheme 1. The synthetic route for synthesis of azide-terminated poly(methyl methacrylate) PMMA via RAFT polymerization.

\subsection{Alkyne-Modification of MWCNT (MWCNTs-alkyne)}

In total, $2 \mathrm{~g}$ of the MWCNTs was subjected to acid treatment with $60 \mathrm{ml}$ of an $\mathrm{HNO}_{3}: \mathrm{H}_{2} \mathrm{SO}_{4}(1: 3)$ mixture using ultrasound at $60{ }^{\circ} \mathrm{C}$ for $4 \mathrm{~h}$, and then refluxed at $80^{\circ} \mathrm{C}$ for $12 \mathrm{~h}$. After the reaction, the treated MWCNTs (MWCNTs-COOH) were washed until the excess acid was completely removed.

In total, $1 \mathrm{~g}$ of MWCNTs-COOH was dispersed in thionyl chloride $(65 \mathrm{~mL})$ for $30 \mathrm{~min}$. Then, $2 \mathrm{ml}$ $\mathrm{N}, \mathrm{N}$-dimethylformamide (DMF) was added to this reaction mixture and stirred for $24 \mathrm{~h}$ at $70{ }^{\circ} \mathrm{C}$. Thus, the MWCNT-COCl was obtained after being dried at $50{ }^{\circ} \mathrm{C}$ for $24 \mathrm{~h}$. After that, $0.5 \mathrm{~g}$ MWCNT-COCl and $2 \mathrm{ml}$ anhydrous triethylamine were mixed in $20 \mathrm{~mL}$ of trichloromethane. Following this, $3 \mathrm{ml}$ of propargyl alcohol was added dropwise to the MWCNT-COCl mixture at $0{ }^{\circ} \mathrm{C}$. The reaction between MWCNT-COCl and propargyl alcohol was carried out at room temperature for $10 \mathrm{~h}$. Subsequently, the obtained MWCNTs-alkyne was purified by centrifugation and then dried at $50{ }^{\circ} \mathrm{C}$ in a vacuum oven for $24 \mathrm{~h}$.

\subsection{Preparation of $M W C N T s-g-P M M A$}

In total, $0.1 \mathrm{~g}$ of MWCNTs-alkyne and $1 \mathrm{~g}$ of azide-terminated PMMA were mixed with $15 \mathrm{~mL}$ of DMF under ultrasonic treatment for $30 \mathrm{~min}$. Then, a CuBr solution ( $0.0069 \mathrm{~g}$ dissolved $1 \mathrm{~mL}$ of water) was added. The Schlenk flask was degassed and back-filled with nitrogen, and then put in an oil bath at $50{ }^{\circ} \mathrm{C}$. After the click reaction, MWCNTs-g-PMMA was purified by ethylenediaminetetraacetic acid (EDTA), THF, and ethanol centrifugation. Unreacted PMMA was removed with THF for $24 \mathrm{~h}$ by 
Soxhlet apparatus and dried for $24 \mathrm{~h}$ in a vacuum oven at $50{ }^{\circ} \mathrm{C}$. The synthesis steps from MWCNTs to MWCNTs-g-PMMA are shown in Scheme 2.

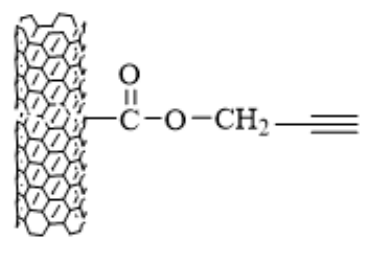

Short-MWCNT-Alkyne

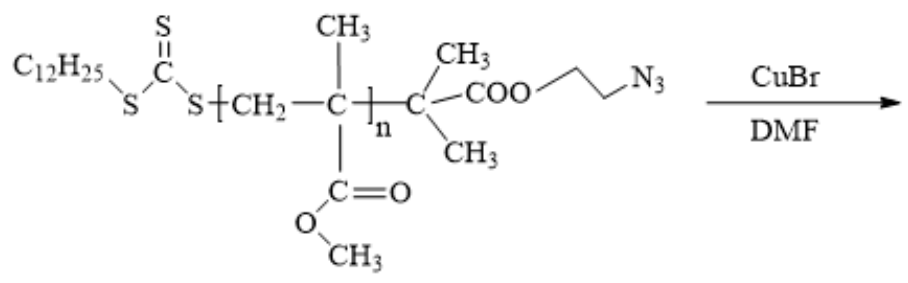

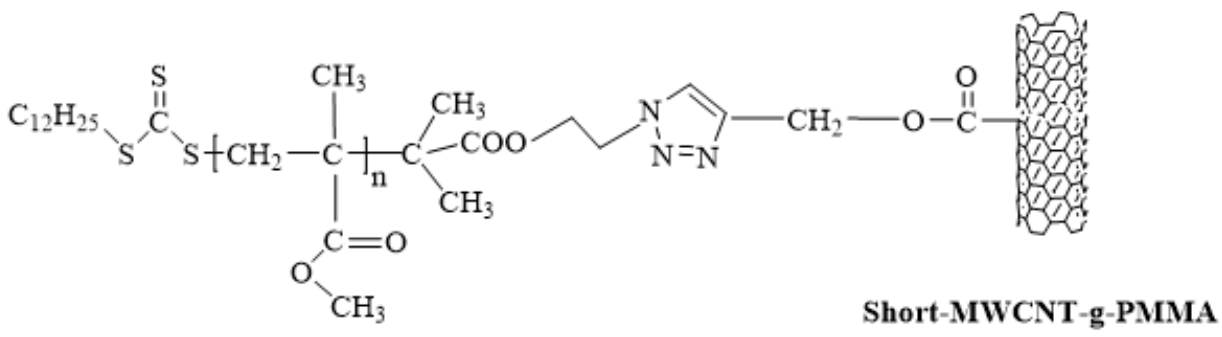

Scheme 2. Synthetic steps from multiwalled carbon nanotube (MWCNT) to MWCNT- $g$-PMMA.

\subsection{Characterization}

Fourier transform infrared spectroscopy (FTIR) spectra were performed on an Avatar 370 spectrometer (Nicolet, USA). The KBr pellet within an appropriate amount of MWCNTs was prepared. Raman spectra of the MWCNTs were gauged using a DXR Raman spectrometer (Thermo Scientific, USA) with the excitation wavelength of the laser at $532 \mathrm{~nm}$. A laser intensity of $7.0 \mathrm{~mW}$, an exposure time of $3 \mathrm{~s}$, and the exposure rate of 20 times were applied in each measurement. An HP-6890 gas chromatograph (GC, Agilent, USA) measured the monomer conversion. Waters 515 gel permeation chromatography (GPC) was equipped with three columns (average pore sizes of 104, 105, and $106 \mathrm{~nm}$, monodisperse polystyrene was used for the calibration standard sample). A Waters RI detector at $35{ }^{\circ} \mathrm{C}$ measured the molecular weight and molecular polydispersity of azide-terminated PMMA. Thermogravimetric analysis (TGA) was performed on a 209 F3 thermogravimetric (TG) analyzer (Netzsch Inc., Germany) under $\mathrm{N}_{2}$ protection with a flow rate of $50 \mathrm{~mL} / \mathrm{min}$. The sample was heated from $50{ }^{\circ} \mathrm{C}$ to $700{ }^{\circ} \mathrm{C}$ at $10{ }^{\circ} \mathrm{C} / \mathrm{min}$. The grafting percentage (GP) for MWCNTs- $g$-PMMA can be calculated as shown in our previous work [24]. Transmission electron microscopy (TEM) was performed on a JEM-1200 EX/S transmission electron microscope (JEOL, Japan). Before observation, the dried MWCNTs were pretreated in THF under ultrasonic vibration for $20 \mathrm{~min}$ and then deposited on a covered copper grid.

\section{Results and Discussions}

\subsection{RAFT Polymerization of Azide-Terminated PMMA}

To investigate the efficiency of PMMA chain end transformation, polymerization kinetic studies on the linear PMMA synthesis were executed. The polymerization progress was checked by taking samples from the reaction mixture, which were measured by GPC and GC to estimate the evolution of conversion, molecular weights, and polydispersity index (PDI) with time. As it can be seen from Figure 2, $\mathrm{M}_{\mathrm{n}}$ is increasing with conversion and PMMA has a small PDI, demonstrating a well-controlled RAFT reaction when using the RAFT agent. At the same time, when the high molecular weight is achieved at a high monomer conversion, the PDI values are still low (around 1.42). Compared with previous work [24], the conversion reaches a higher level ( 55\%), and the $\mathrm{M}_{\mathrm{n}}$ of azide-terminated 
PMMA increases more quickly with conversion, which suggests a much quicker reaction rate in this work.

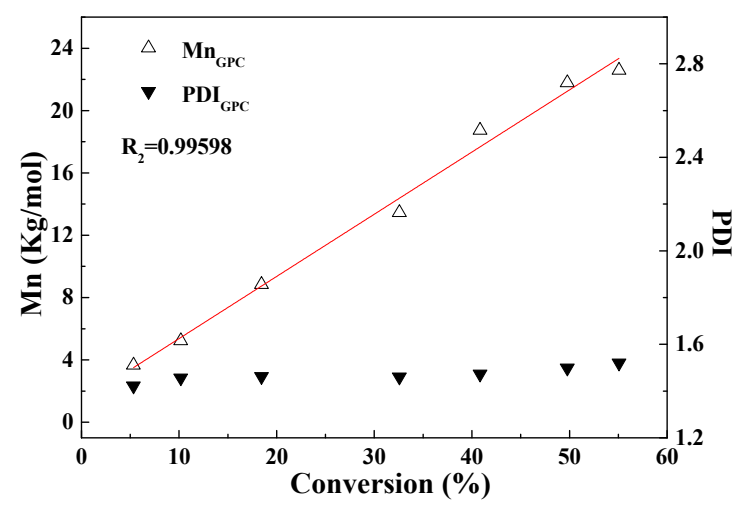

Figure 2. Dependence of number-average molecular weight $\left(M_{n}\right)$ and PDI $\left(M_{W} / M_{n}\right)$ of the grafted PMMA on the conversion for the PMMA RAFT polymerization at $60^{\circ} \mathrm{C}$ with AIBN as initiator mediated by DMP-N ${ }_{3}\left([\mathrm{MMA}]_{0}:[\mathrm{RAFT}]_{0}:[\mathrm{AIBN}]_{0}=250: 1: 0.2\right)$.

The results on the RAFT polymerization of the azide-terminated PMMA show a linear increase in $\ln \left(\mathrm{M}_{0} / \mathrm{M}_{\mathrm{t}}\right)$ with time (Figure 3), suggesting a constant radical concentration, i.e., the absence of extensive termination reactions [25]. A conversion of $55 \%$ was obtained in $10 \mathrm{~h}$, resulting in an azide-terminated PMMA with $\mathrm{M}_{\mathrm{n}}=22000 \mathrm{~g} / \mathrm{mol}$ and PDI $=1.50$.

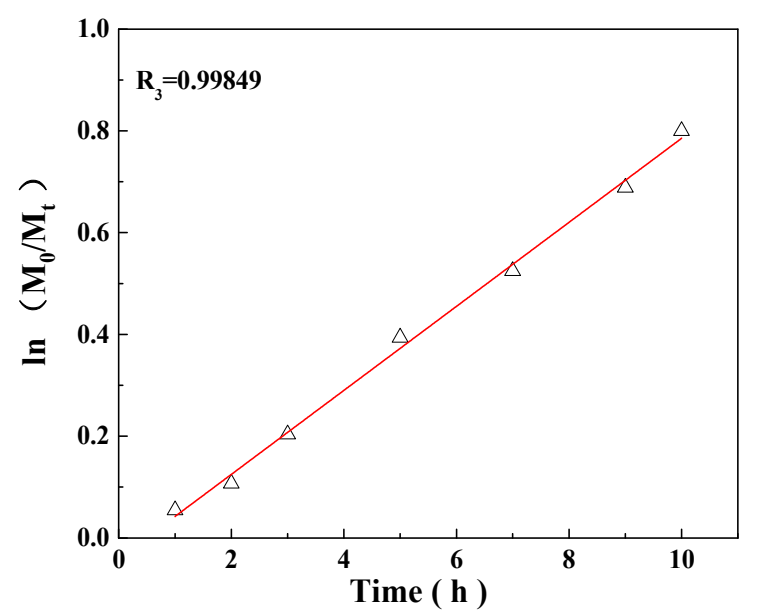

Figure 3. First-order kinetic plot for the RAFT polymerization of PMMA at $60{ }^{\circ} \mathrm{C}$ with $\mathrm{AIBN}$ as initiator mediated by DMP-N $3\left([\mathrm{MMA}]_{0}:[\mathrm{RAFT}]_{0}:[\mathrm{AIBN}]_{0}=250: 1: 0.2\right)$.

In general, the MWCNTs-g-PMMA was obtained through the click coupling between MWCNTs-alkyne and azide-terminated PMMA. The grafting PMMA molecular weight was controlled by the prior synthesis of azide-terminated PMMA via RAFT polymerization. The grafting percentage on the MWCNT surface can present the click reaction efficiency with the reaction time. In this work, TGA measurement elucidated the grafting percentage that governed the alkyne/azide click reaction on MWCNT surfaces, as shown in Figure 4. It is evident that the grafting rate of PMMA on the surface of MWCNTs rises with the increase of reaction time. The grafting rate of MWCNTs-g-PMMA reaches $21.9 \%$ after a reaction of $24 \mathrm{~h}$, and when the reaction continues to $30 \mathrm{~h}$, the grafting rate is not changed obviously. The saturation grafting rate after $24 \mathrm{~h}$ could be due to the steric demands of the clicked PMMA chains rendering the remaining azido groups inaccessible for the click reaction [26]. 


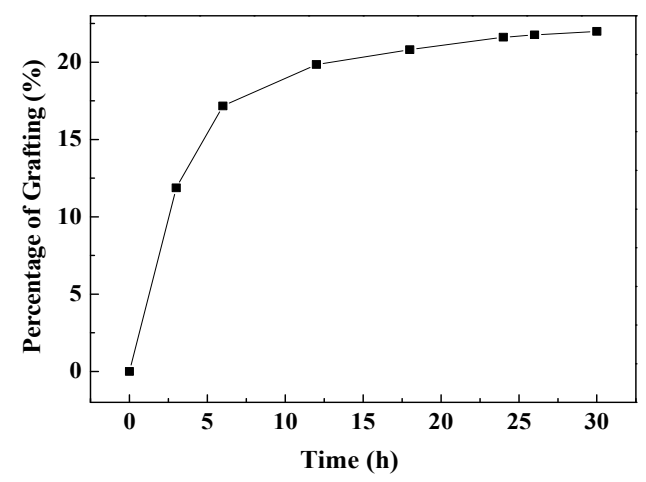

Figure 4. Grafting percentage of the azide/alkyne modified MWCNT.

\subsection{TGA Analysis for the MWCNTS-g-PMMA}

The TGA curves of pristine MWCNTs, MWCNTs-alkyne, and MWCNTs- $g$-PMMA are presented in Figure 5. Two stages of significant mass losses are observed in the curves of pristine MWCNTs. The first weight loss of about $0.5 \%$ happens before $200{ }^{\circ} \mathrm{C}$, which is due to the loss of adsorbed water. The second step at a weight loss of approximately $1.4 \%$ is due to the impurities of pyrolysis. For the MWCNTs- alkyne, because of the decomposition of organic groups on the surface, the final weight loss was increased to $5.27 \%$. For MWCNTs-g-PMMAs received at various polymerization times during the click reactions, because the grafted PMMA chains decompose to different extents, the final weight losses for the reaction time at $6 \mathrm{~h}, 12 \mathrm{~h}$, and $18 \mathrm{~h}$ are $22.4 \%, 25.1 \%$, and $26.9 \%$, respectively (Figure $5 \mathrm{c}-\mathrm{e}$ ). The curves for the MWCNT-g-PMMA show two main decomposition steps at $205^{\circ} \mathrm{C}$ and $405^{\circ} \mathrm{C}$, which correspond to the side chains and PMMA chains respectively. As measured, the grafting percentage (GP) for a reaction time of $6 \mathrm{~h}, 12 \mathrm{~h}$, and $18 \mathrm{~h}$ is $17.2 \%, 19.9 \%$, and $22.0 \%$, respectively.

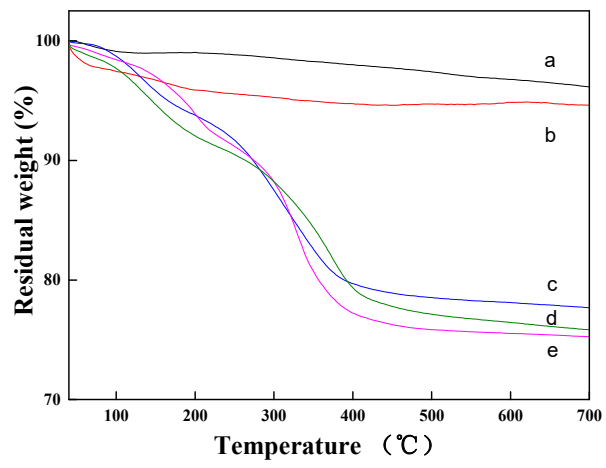

Figure 5. TGA results of pristine MWCNTs (a) MWCNTs-alkyne (b) MWCNTs-g-PMMA (c-e) polymerization for 6,12 , and $18 \mathrm{~h}$, respectively.

\subsection{Surface Structure Analysis for MWCNTS}

The MWCNTs-alkyne was obtained via two gentle reaction processes, including acylchlorination and esterification, during which MWCNT-COOH was reacted with excess thionyl chloride to obtain a high esterification reaction efficiency COCL group, and the COCL group was then reacted with excess propargyl alcohol to get a complete surface coverage of the functional MWCNTs. FT-IR was performed on original and functionalized MWCNTs, and their corresponding spectra are shown in Figure 6. For the MWCNTs-COOH, the IR spectrum shows two absorption bands at $1740 \mathrm{~cm}^{-1}$ (corresponding to stretching vibrations of carbonyl groups $\mathrm{C}=\mathrm{O}$ ) and $1635 \mathrm{~cm}^{-1}$ (assigned to conjugated $\mathrm{C}=\mathrm{C}$ stretching). MWCNTs-alkyne exhibits the same bands with the addition of an intensity band at $2100 \mathrm{~cm}^{-1}$ (the alkyne group) [27]. Azido-terminated PMMA presents a typical absorption band at $2100 \mathrm{~cm}^{-1}$ (-N $\mathrm{N}_{3}$ group), which suggests that the subsequent click reaction can be performed. After the click reaction, the new absorptions peak appeared at $1110 \mathrm{~cm}^{-1}$, and $1020 \mathrm{~cm}^{-1}$, which was the $\mathrm{C}-\mathrm{O}-\mathrm{C}$ 
group in the ester group of the PMMA grafted onto the surface of MWCNTs. The click combination of the alkyne-functionalized MWCNTs and azide-functionalized PMMA provided a 1,2,3-triazole ring. This suggests that the PMMA molecule is successfully grafted onto the surface of the MWCNTs. Thus, the IR spectra of the MWCNTs-PMMA nanohybrid, featuring an alkyne peak of MWCNTs at $2100 \mathrm{~cm}^{-1}$, entirely disappeared, revealing the formation of 1,2,3-triazole after the click reaction.

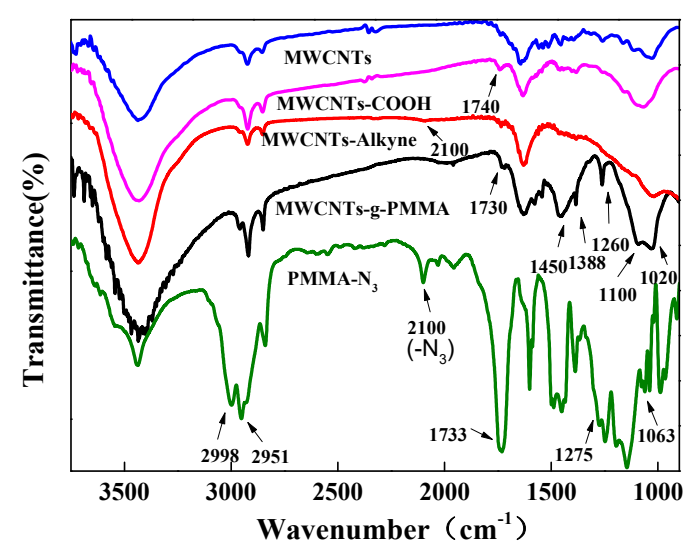

Figure 6. FT-IR spectra of pristine MWCNTs, MWCNTs-COOH, MWCNTs-alkyne, PMMA-N 3 , and MWCNTs- $g$-PMMA (GP = 22.0\%).

Figure 7 shows the Raman spectra of pristine MWCNTs, MWCNTs-COOH, MWCNTs-alkyne, and MWCNTs-g-PMMA. The characteristic peaks at $1343 \mathrm{~cm}^{-1}$ and $1585 \mathrm{~cm}^{-1}$ correspond to the D (tangential band) and G (disorder band) peaks of carbon nanotubes, respectively [22]. The D band is due to a disordered graphite structure or $\mathrm{sp}^{3}$-hybridized carbons of the nanotubes, whereas the $\mathrm{G}$ band refers to a splitting of the E2g stretching mode of graphite $[28,29]$. The peak intensity D and G band ratios $\left(\mathrm{I}_{\mathrm{D}} / \mathrm{I}_{\mathrm{G}}\right)$ for MWCNTs-COOH, MWCNTs-alkyne, and MWCNTs-g-PMMA are higher than those of the pristine MWCNTs, which suggests that alkyne-decoration and the click reaction successfully functionalize MWCNT.

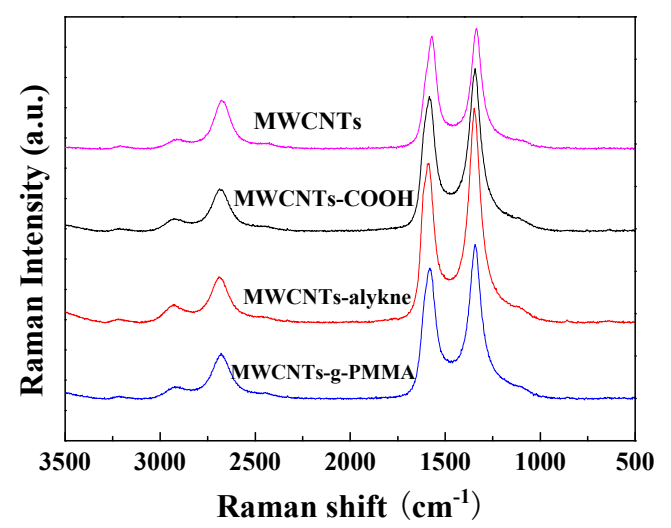

Figure 7. Raman spectra of pristine MWCNT, MWCNTs-COOH, MWCNTs-alkyne, and MWCNTs-gPMMA $(\mathrm{GP}=22.0 \%)$.

Figure 8 depicts the TEM photographs of pristine MWCNTs, carboxyl functionalized, and MWCNTs- $g$-PMMA. After the acid treatment, the closed end opening of the nanotube can be observed. The pristine MWCNTs generally present closed caps and cylindrical walls, which are uncapped and have rough "convex-concave" walls after the partial oxidation treatment [30]. These disfigurements improve the specific surface area and pore volume of the oxidized MWCNTs [31]. On the other hand, after the PMMA segments are grafted onto the surface of nanotubes by the click reaction, a thicker diameter of the MWCNTs-g-PMMA is observed. 

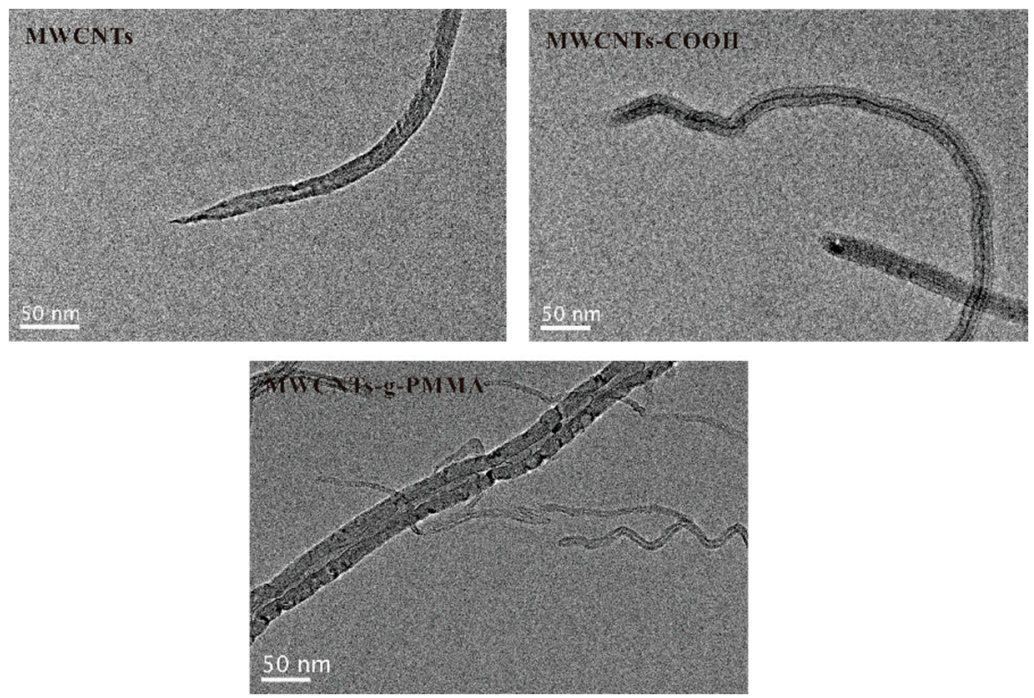

Figure 8. TEM photographs of the pristine MWCNTs, MWCNT-COOH, and MWCNTs- $g$-PMMA (GP $=22.0 \%$ ).

Figure 9 shows the dispersion images of MWCNTs-g-PMMA in comparison with the pristine MWCNTs in water. After the ultrasonic treatment of these two dispersing solutions, the pristine MWCNTs could not disperse and stably suspended well in water because of the strong intrinsic van der Waals forces between them [13]. After two hours of standing, the pristine MWCNTs are obviously aggregated. However, the MWCNTs-g-PMMA can maintain its stable dispersion, even after 24 hours. This means that grafted PMMA has effectively reduced the apparent activation energy of MWCNTs, which can prevent the aggregation phenomenon. In future work, we will study this MWCNTs-g-PMMA dispersion in the polymeric membrane bulk.

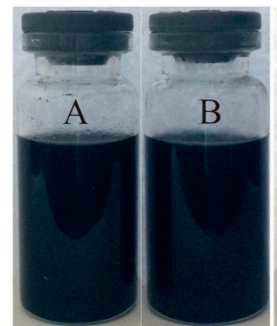

(a)

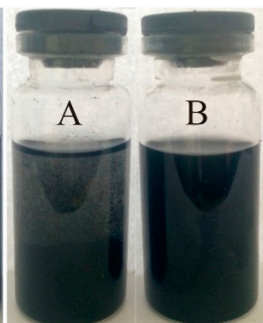

(b)

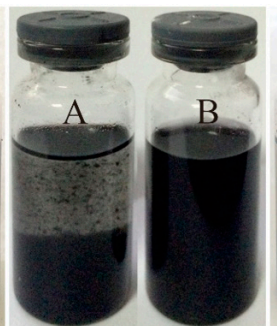

(c)

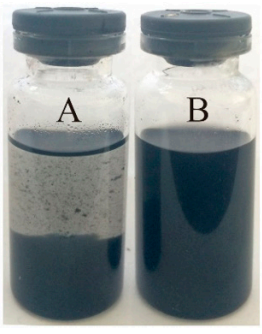

(d)

Figure 9. The dispersion images of pristine MWCNTs (A) and MWCNTs- $g$-PMMA (GP = 22.0\%) (B) in water. (a): after 0 h; (b): after 1 h; (c): after 2 h; (d): after $24 \mathrm{~h}$.

\section{Conclusions}

In this work, to avoid the aggregation of MWCNTs, well-defined PMMA functionalized the combination of RAFT synthesized MWCNTs (MWCNTs-g-PMMA) and the clicked reaction. The success of PMMA grafting onto MWCNTs was determined by GPC, Raman spectroscopy, FT-IR, TGA, and TEM measurements. The kinetic reaction results show that the grafting percentage of PMMA chains grafted onto the MWCNTs surface rises with the increase of reaction time. Even at a low temperature $\left(50^{\circ} \mathrm{C}\right)$, the grafting rate of azide-terminated PMMA is comparatively fast during the click reaction when combining the alkyne-MWCNTs and azide-terminated PMMA in less than $24 \mathrm{~h}$. As calculated by TGA analysis, the highest grafting degree of PMMA chains reaches $21.9 \%$. Compared with the pristine MWCNTs, a thicker diameter of the MWCNTs-g-PMMA was observed by TEM, which confirmed that the PMMA chain grafted onto the surface of nanotubes. Therefore, the MWCNTs- $g$-PMMA could be dispersed and stably suspended in water, even for $24 \mathrm{~h}$. 
Author Contributions: W.M. conceived the experiments and wrote the manuscript; Y.Z. designed the experiments; Z.Z. and L.G. performed the click reaction experiments; Z.C. and Y.X. contributed to the results and discussions; H.Y. and F.G. gave the RAFT synthesis methods; J.Z. reviewed this manuscript and checked the English language corrections.

Funding: This research was funded by the Natural Science Foundation of China (Grant No. 21406017); the Natural Science Foundation of Jiangsu Province, China (Grant No. BK20140254); the Natural Science Foundation of the Jiangsu Higher Institutions of China (18KJA430005).

Acknowledgments: Thanks to the Priority Academic Program Development of Jiangsu Higher Education Institutions (PAPD) support.

Conflicts of Interest: The authors declare no conflict of interest.

\section{References}

1. Qu, X.L.; Alvarez, P.J.J.; Li, Q.L. Applications of nanotechnology in water and wastewater treatment. Water Resour. 2013, 47, 3931-3946. [CrossRef] [PubMed]

2. Bell, A.T. The impact of nanoscience on heterogeneous catalysis. Science 2003, 299, 1688-1691. [CrossRef] [PubMed]

3. Hummer, G.; Rasaiah, J.C.; Noworyta, J.P. Water conduction through the hydrophobic channel of a carbon nanotube. Nature 2001, 414, 188-190. [CrossRef] [PubMed]

4. Waghe, A.; Rasaiah, J.C.; Hummer, G. Filling and emptying kinetics of carbon nanotubes in water. J. Chem. Phys. 2002, 117, 10789-10795. [CrossRef]

5. Holt, J.K.; Park, H.G.; Wang, Y.M.; Stadermann, M.; Artyukhin, A.B.; Grigoropoulos, C.P.; Noy, A.; Bakajin, O. Fast mass transport through sub-2-nanometer carbon nanotubes. Science 2006, 312, 1034-1037. [CrossRef] [PubMed]

6. Majumder, M.; Chopra, N.; Andrews, R.; Hinds, B.J. Nanoscale hydrodynamics-Enhanced flow in carbon nanotubes. Nature 2005, 438, 44. [CrossRef]

7. Tunuguntla, R.H.; Henley, R.Y.; Yao, Y.C.; Pham, T.A.; Wanunu, M.; Noy, A. Enhanced water permeability and tunable ion selectivity in subnanometer carbon nanotube porins. Science 2017, 357, 792-796. [CrossRef]

8. Liu, Z.P.; Yang, R. Synergistically-enhanced thermal conductivity of shape-stabilized phase change materials by expanded graphite and carbon nanotube. Appl. Sci. 2017, 7, 574. [CrossRef]

9. Ma, W.; Gong, F.; Liu, C.; Tao, G.; Xu, J.; Jiang, B. SiO 2 reinforced HDPE hybrid materials obtained by the sol-gel method. J. Appl. Polym. Sci. 2014, 131, 596-602. [CrossRef]

10. Giovino, M.; Pribyl, J.; Benicewicz, B.; Kumar, S.; Schadler, L. Linear rheology of polymer nanocomposites with polymer-grafted nanoparticles. Polymer 2017, 131, 104-110. [CrossRef]

11. Fontananova, E.; Bahattab, M.A.; Aljlil, S.A.; Alowairdy, M.; Rinaldi, G.; Vuono, D.; Nagy, J.B.; Drioli, E.; Di Profio, G. From hydrophobic to hydrophilic polyvinylidenefluoride (PVDF) membranes by gaining new insight into material's properties. RSC Adv. 2015, 5, 56219-56231. [CrossRef]

12. Li, H.B.; Shi, W.Y.; Su, Y.H.; Zhang, H.X.; Qin, X.H. Preparation and characterization of carboxylated multiwalled carbon nanotube/polyamide composite nanofiltration membranes with improved performance. J. Appl. Polym. Sci. 2017, 134, e45268. [CrossRef]

13. Sanip, S.M.; Ismail, A.F.; Goh, P.S.; Soga, T.; Tanemura, M.; Yasuhiko, H. Gas separation properties of functionalized carbon nanotubes mixed matrix membranes. Sep. Purif. Technol. 2011, 78, 208-213. [CrossRef]

14. Balazs, A.C.; Emrick, T.; Russell, T.P. Nanoparticle polymer composites: Where two small worlds meet. Science 2006, 314, 1107-1110. [CrossRef] [PubMed]

15. Zdyrko, B.; Luzinov, I. Polymer brushes by the "grafting to" method. Macromol. Rapid. Comm. 2011, 32, 859-869. [CrossRef] [PubMed]

16. Ma, W.; Zhou, B.; Liu, T.; Zhang, J.; Wang, X. The supramolecular organization of PVDF lamellae formed in diphenyl ketone dilutions via thermally induced phase separation. Colloid Polym. Sci. 2013, 291, 981-992. [CrossRef]

17. Wu, P.; Feldman, A.K.; Nugent, A.K.; Hawker, C.J.; Scheel, A.; Voit, B.; Pyun, J.; Frechet, J.M.J.; Sharpless, K.B.; Fokin, V.V. Efficiency and fidelity in a click-chemistry route to triazole dendrimers by the copper(I)-catalyzed ligation of azides and alkynes. Angew. Chem. Int. Ed. 2004, 43, 3928-3932. [CrossRef] [PubMed]

18. Binder, W.H.; Sachsenhofer, R. 'Click' chemistry in polymer and materials science. Macromol. Rapid Commun. 2007, 28, 15-54. [CrossRef] 
19. Escorihuela, J.; Marcelis, A.T.; Zuilhof, H. Metal-free click chemistry reactions on surfaces. Adv. Mater. Interfaces 2015, 2, 1500135.

20. Barner-Kowollik, C.; Du Prez, F.E.; Espeel, P.; Hawker, C.J.; Junkers, T.; Schlaad, H.; Camp, W.V. “Clicking” polymers or just efficient linking: What is the difference? Ang. Chem. Int. Ed. 2011, 50, 60-62. [CrossRef]

21. Zhao, L.J.; Zhao, F.Q.; Zeng, B.Z. Synthesis of water-compatible surface-imprinted polymer via click chemistry and RAFT precipitation polymerization for highly selective and sensitive electrochemical assay of fenitrothion. Biosens. Bioelectron. 2014, 62, 19-24. [CrossRef] [PubMed]

22. Pramanik, N.B.; Singha, N.K. Direct functionalization of multi-walled carbon nanotubes (MWCNTs) via grafting of poly(furfuryl methacrylate) using Diels-Alder "click chemistry" and its thermoreversibility. RSC Adv. 2015, 5, 94321-94327. [CrossRef]

23. Gondi, S.R.; Vogt, A.P.; Sumerlin, B.S. Versatile pathway to functional telechelics via RAFT polymerization and click chemistry. Macromolecules 2007, 40, 474-481. [CrossRef]

24. Ma, W.Z.; Zhao, Y.C.; Li, Y.X.; Zhang, P.; Cao, Z.; Yang, H.C.; Liu, C.L.; Tao, G.L.; Gong, F.H.; Matsuyama, H. Synthesis of hydrophilic carbon nanotubes by grafting poly(methyl methacrylate) via click reaction and its effect on poly(vinylidene fluoride)-carbon nanotube composite membrane properties. Appl. Surf. Sci. 2018, 435, 79-90. [CrossRef]

25. Can, A.; Altuntas, E.; Hoogenboom, R.; Schubert, U.S. Synthesis and MALDI-TOF-MS of PS-PMA and PMA-PS block copolymers. Eur. Polym. J. 2010, 46, 1932-1939. [CrossRef]

26. Chen, J.C.; Liu, M.Z.; Chen, C.; Gong, H.H.; Gao, C.M. Synthesis and characterization of silica nanoparticles with well-defined thermoresponsive PNIPAM via a combination of RAFT and click chemistry. ACS Appl. Mater. Interfaces 2011, 3, 3215-3223. [CrossRef] [PubMed]

27. Chang, Z.J.; Xu, Y.; Zhao, X.; Zhang, Q.H.; Chen, D.J. Grafting poly(methyl methacrylate) onto polyimide nanofibers via "click" reaction. ACS Appl. Mater. Interfaces 2009, 1, 2804-2811. [CrossRef] [PubMed]

28. Osswald, S.; Havel, M.; Gogotsi, Y. Monitoring oxidation of multiwalled carbon nanotubes by Raman spectroscopy. J. Raman Spectrosc. 2007, 38, 728-736. [CrossRef]

29. Jorio, A.; Dresselhaus, G.; Dresselhaus, M.S.; Souza, M.; Dantas, M.S.S.; Pimenta, M.A.; Rao, A.M.; Saito, R.; Liu, C.; Cheng, H.M. Polarized Raman study of single-wall semiconducting carbon nanotubes. Phys. Rev. Lett. 2000, 85, 2617-2620. [CrossRef]

30. Svrcek, V.; Pham-Huu, C.; Amadou, J.; Begin, D.; Ledoux, M.-J.; Le Normand, F.; Ersen, O.; Joulie, S. Filling and capping multiwall carbon nanotubes with silicon nanocrystals dispersed in $\mathrm{SiO} 2-$ based spin on glass. J. Appl. Phys. 2006, 99, 064306. [CrossRef]

31. Hu, C.C.; Su, J.H.; Wen, T.C. Modification of multi-walled carbon nanotubes for electric double-layer capacitors: Tube opening and surface functionalization. J. Phys. Chem. Solids 2007, 68, 2353-2362. [CrossRef] 